\title{
Modeling and Optimization of RFID Networks Planning Problem
}

\author{
Hongshan Kong $(\mathbb{D}$ and Bin Yu \\ Information Engineering University, Zhengzhou, China \\ Correspondence should be addressed to Hongshan Kong; m13643861930@163.com
}

Received 28 May 2019; Accepted 15 November 2019; Published 7 December 2019

Guest Editor: Dionisis Kandris

Copyright $\odot 2019$ Hongshan Kong and Bin Yu. This is an open access article distributed under the Creative Commons Attribution License, which permits unrestricted use, distribution, and reproduction in any medium, provided the original work is properly cited.

\begin{abstract}
Aimed at solving the RFID networks planning problem, a mathematical model considering tag coverage and reader interference is presented. The DEEPSO algorithm that adds differential evolution and evolutionary strategies to the standard PSO is introduced to the optimization of RFID Networks Planning, which can improve the global convergence ability and particle diversity and can avoid falling into local convergence. According to the simulation results, compared with RFID networks planning by standard PSO, RFID networks planning by DEEPSO is superior.
\end{abstract}

\section{Introduction}

Radio frequency identification (RFID) technology has been widely applied to asset tracking, smart grid, car manufacturing, and supply chain management. The basic components of RFID system are tags and readers. Readers gain access to the information stored on the tags within a distance of several meters [1]. Then, readers and tags need to be deployed in the working area, which must consider several practical problems, such as the tag coverage, the cost efficiency, and the quality of service (QoS) [2]. The RFID network planning (RNP) problem aims to optimize a set of objectives by adjusting the control variables of the system and RNP is a difficult NP problem [3]. Because RNP is a large-scale, highdimensional nonlinear optimization problem with large number of uncertain parameters, it cannot be tackled by the traditional techniques [4].

Metaheuristic is an efficient approach for hard optimization problems. For solving the RNP problem, evolutionary computation and swarm intelligence techniques have been applied, such as genetic algorithms [5], evolutionary strategy [6], particle swarm optimization (PSO) algorithms [7, 8], firefly algorithms [9], artificial bee colony algorithms [2], and bacterial foraging algorithms [10]. However, with the increasing number of the deployed readers and tags in the large-scale RFID deployment environment, the degree of complexity for solving the RNP optimization increases exponentially. The previous methods to solve the RNP optimization are incompetent for being prone to premature convergence.

For avoiding premature convergence, the idea of heuristic algorithm has been developed into an important and effective way to improve the optimization performance, which can combine the advantages of various algorithms. In [11], a hybrid algorithm combining genetic algorithm and particle swarm optimization is proposed and applied RNP optimization. In [12], a hybrid algorithm combining genetic algorithm and artificial bee colony algorithm is proposed and applied RNP optimization. DEEPSO [13] is a hybrid algorithm based on PSO combining differential evolution and evolutionary computation, which was the winner in 2014 of the competition on the application of modern heuristic optimization algorithms for solving optimal power flow problems [14]. For increasing the optimization performance of RFID Networks Planning, the DEEPSO algorithm is introduced and applied.

The rest of the paper is organized as follows. In Section 2, RFID network planning problem and Mathematical model are presented. Section 3 first gives a review of the DEEPSO algorithm and then proposes the realization method of RFID network planning problem using DEEPSO. In Section 4, the simulation experiments and results analysis of RFID network planning problem using DEEPSO are presented. Finally, Section 5 outlines the conclusions. 


\section{RFID Network Planning Problem and Modeling}

2.1. Problem Description. The key components of an RFID system are the tags and readers. The RFID tag, which is attached to the item to be tracked, stores the unique identification number of the item using a small integrated circuit. The RFID readers communicate with the tags by reading the information stored on them. The reader has a limit on its interrogation range, within which the tags can be read. Thus, the RFID network planning problem is a difficult problem that needs to be solved in order to deploy and operate the large-scale network of RFID readers in an optimal fashion. Consider the following:

(1) The monitoring area is a two-dimensional plane

(2) An appropriate amount of tags are randomly placed in the area

(3) The coverage of the reader is a circular area centered

(4) The readers are isomorphic with the same perceived radius

(5) The reader can be moved and the energy is sufficient to ensure that the final positional movement is completed

2.2. Mathematical Model. The symbols and declarations of the mathematical model are shown in Table 1.

The first objective function $f_{c}$ represents the tag coverage, which is the most important in an RFID system. If the distance between the $i^{\text {th }}$ tag and the $k^{\text {th }}$ reader is smaller than the sensing radius of reader, the communication between the $i^{\text {th }}$ tag and the $k^{\text {th }}$ reader can be established. Then the function is formulated as

$$
\max f_{c}=\frac{1}{N_{t}} \cdot \sum_{i=1}^{N_{t}} \sum_{k=1}^{N_{r}} p_{1}\left(t_{i}, r_{k}\right),
$$

where

$$
p_{1}\left(t_{i}, r_{k}\right)=\left\{\begin{array}{l}
1, \text { if } d\left(t_{i}, r_{k}\right) \leq R, \\
0, \text { else. }
\end{array}\right.
$$

The second objective function $f_{i}$ represents the reader interference. Reader collision mainly occurs in a dense reader environment, where several readers try to interrogate tags at the same time, which results in an unacceptable level of misreads. The objective function is formulated as

$$
\min f_{i}=\frac{1}{N_{t}} \cdot \sum_{i=1}^{N_{t}} p_{2}\left(t_{i}\right),
$$

where

$$
p_{2}\left(t_{i}\right)= \begin{cases}1, & \text { if } \sum_{k=1}^{N_{t}} p_{1}\left(t_{i}, r_{k}\right) \geq 2, \\ 0, & \text { else. }\end{cases}
$$

In order to align with the first objective optimization direction, modify (3) into the following formula:
TABLE 1: The symbols and declarations.

\begin{tabular}{lc}
\hline Symbol & Declaration \\
\hline$N_{t}$ & The number of tags \\
$t_{i}$ & The coordinates of $i^{\text {th }}$ tag, $t_{i}=\left(x t_{i}, y t_{i}\right)$ \\
$N_{r}$ & The number of readers \\
$r_{k}$ & The coordinates of $k^{\text {th }}$ reader, $r_{k}=\left(x r_{k}, y r_{k}\right)$ \\
$R$ & The sensing radius of reader \\
$d\left(t_{i}, r_{k}\right)$ & The distance between the $i^{\text {th }}$ tag and the $k^{\text {th }}$ reader \\
\hline
\end{tabular}

$$
\max f_{i}^{\prime}=\frac{1}{\sum_{i=1}^{N_{t}} p_{2}\left(t_{i}\right)+1}
$$

Then, the RNP problem concerns the tag coverage and the reader interference that are formulated as follows:

$$
\left\{\begin{array}{l}
\max f=w_{c} f_{c}+w_{i} f_{i}^{\prime}, \\
w_{c}+w_{i}=1 .
\end{array}\right.
$$

\section{RFID Network Planning Optimization Based on DEEPSO}

3.1. DEEPSO. Assumed in the space $D$, randomly initialize a population of particles $P=\left\{p_{1}, p_{2}, \ldots, p_{N}\right\}$ with number of particles $N$. The position and velocity of the $i$ particle are $x_{i}=\left(x_{i 1}, x_{i 2}, \ldots, x_{i D}\right)^{T}$ and $v_{i}=\left(v_{i 1}, v_{i 2}, \ldots, v_{i D}\right)^{T}$. The individual extremum of the population is expressed as best $_{i}=\left(\text { best }_{i 1} \text {, best }_{i 2}, \ldots \text {, best }_{i D}\right)^{T}$, the global extremum is expressed as gbest $=\left(\text { gbest }_{1}, \text { gbest }_{2}, \ldots, \text { gbest }_{D}\right)^{T}$, particles update speed, and position according to the following formula:

$$
\begin{aligned}
v^{(k+1)}= & w v_{i}^{(k)}+c_{1} \cdot \operatorname{rand}() \cdot\left(\text { best }_{i}^{(k)}-x_{i}^{(k)}\right) \\
& +c_{2} \cdot \operatorname{rand}() \cdot\left(g \text { best }-x_{i}^{(k)}\right), \\
x_{i}^{(k+1)}= & x_{i}^{(k)}+v_{i}^{(k)},
\end{aligned}
$$

in which $k$ denotes the current generation, $\operatorname{rand}()$ is a random number between $(0,1), c_{1}$ and $c_{2}$ are acceleration constants, which represent the weight of particles moving toward individual extremum and global extremum, and $w$ is the inertia coefficient, when $w$ is larger, representations have stronger global search capabilities, when $w$ is smaller, representations have stronger local search capabilities.

The improvement of DEEPSO over PSO consists of using a recombination mechanism with Differential Evolution (DE) and using a self-adaptive recombination operator with Evolutionary Strategies (ES). On one hand, DEEPSO relies on the past information of optimization process to create new promising solutions and proposes the replacement of the individual memory by a collective memory to enable an improved sensitivity of the optimization landscape. On the other hand, DEEPSO has self-adaptive properties by a selfadaptive recombination operator. The DEEPSO movement rule based on classical PSO by replacing the memory term by the perception term as 


$$
\begin{aligned}
v^{(k+1)}= & w_{1} \cdot v^{(k)}+w_{2} \cdot\left(\text { best }_{r}^{(k)}-x^{(k)}\right) \\
& +w_{3} \cdot\left[C \cdot\left(\text { gbest }^{*}-x^{(k)}\right)\right],
\end{aligned}
$$

in which $w_{1}, w_{2}$, and $w_{3}$ represent inertia, memory, and cooperation weights, respectively, $C$ is an $N \times N$ diagonal matrix of random variables that follow a Bernoulli distribution with success probability $P$, best ${ }_{r}^{(k)}$ represents an individual from memory $B$ that is computed every iteration according to one strategy of sampling in the same generation and sampling from the past bests, the superscript gbest* indicates that the parameter undergoes evolution under a mutation process:

$$
\text { gbest }^{*}=\text { gbest }+\tau \cdot N(0,1)
$$

where $\tau$ is the mutation rate and $N(0,1)$ is a number sampled from the standard Gaussian distribution.

\subsection{RFID Network Planning Optimization Based on DEEPSO.} When applying the DEEPSO algorithm to RFID network planning optimization, two problems must be solved: parameter coding and fitness function. Randomly deploying $m$ nodes to the monitoring area can be seen as a continuous optimization problem. Each deployment scenario includes $2 m$ elements, which represent the $x$ coordinates and the $y$ coordinates of $m$ nodes. Define the fitness function of particles in the RFID network planning optimization algorithm as equation (6). Suppose the number of particles is $N$, each deployment scenario can be thought of as a particle. The goal of RFID network planning optimization is to find the particle that maximizes the fitness function value.

To using DEEPSO to solve the RNP problem, the following steps should be taken and repeated.

Step 1. Initialization: There are RFID deployment parameters and simulation parameters. The deployment parameters consist of the shape, size, and dimension of the working area, the number of tags $N_{t}$, the coordinates of tags $t_{i}$, the number of readers $N_{r}$, and the sensing radius of reader $R$. Simulation parameters consist of the positions $\left\{x_{k}^{(i)}\right\}_{i=1}^{N}$ and the velocities $\left\{v_{k}^{(i)}\right\}_{i=1}^{N}$ of particles, the inertia weight $w_{1}$, the memory weight $w_{2}$, and the cooperation weight $w_{3}$, the success probability $P$, the mutation rate $\tau$, and the simulation generation Gen.

Step 2. Evaluate: Calculate the fitness function values of all particles using equation (6), get the best individual $g$ best and memory $B$.

Step 3. Loop: Determine whether the generation number is smaller than the simulation generation Gen. If so, firstly, compute the perception term best ${ }_{r}^{(k)}$ for the current individual according to the perception strategy; secondly, copy the current individual; thirdly, mutate the strategic parameters gbest* of the copied individual according to (10); fourthly, move the current individual and its copy according to 8) and (9); lastly, evaluate the current individual and its copy, and update the best individual $g$ best and memory $B$. If not, stop the procedure.

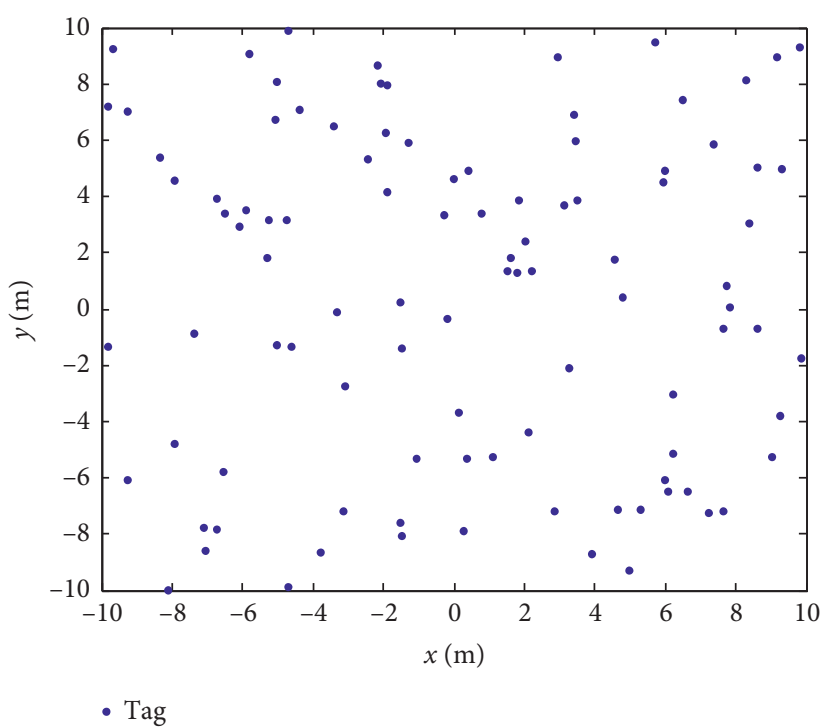

FIgUre 1: The initial placement of square working area.

TABle 2: Simulation parameter setting.

\begin{tabular}{lcc}
\hline Parameters & Setting values & Algorithms \\
\hline$N$ & 50 & PSO and DEEPSO \\
Gen & 150 & PSO and DEEPSO \\
$c_{1}$ & 1.467 & PSO \\
$c_{2}$ & 1.467 & PSO \\
$w$ & 0.729844 & PSO \\
$w_{1}$ & rand () & DEEPSO \\
$w_{2}$ & rand () & DEEPSO \\
$w_{3}$ & rand () & DEEPSO \\
$P$ & 0.8 & DEEPSO \\
$\tau$ & 0.4 & DEEPSO \\
$\omega_{c}$ & 0.8 & DEEPSO \\
$\omega_{i}$ & 0.2 & DEEPSO \\
\hline
\end{tabular}

Step 4. Termination: The results containing the coordinates of readers and the fitness values are given.

\section{Simulation Experiment and Analysis}

In order to measure the optimization performance of the proposed method, the MATLAB software was used to implement numerical simulation.

\subsection{Simulation Experiment Environment and Parameter} Settings. All the algorithms are evaluated against an ideal square working area (a $30 \mathrm{~m} * 30 \mathrm{~m}$ working space with 100 tags that distributed randomly), which is shown in Figure 1.

The RFID reader has a working radius of $3 \mathrm{~m}$ and uses 10 RFID readers to collect data from the tags in the work area. The experiment uses PSO and DEEPSO to optimize the planning of the RFID reader network. The parameters are shown in Table 2.

In the work area, 50 deployment locations are randomly generated for 10 readers, respectively, and are the initial position values of the particles in the particle swarm 


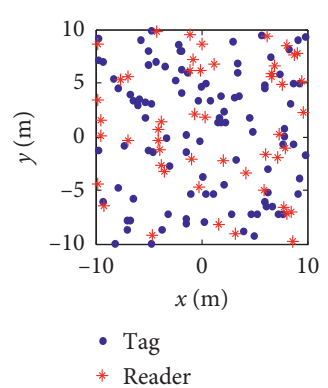

(a)

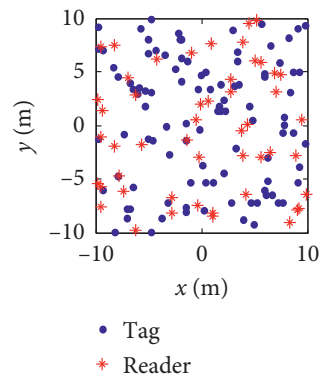

(f)

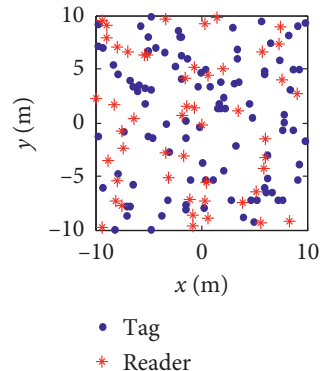

(b)

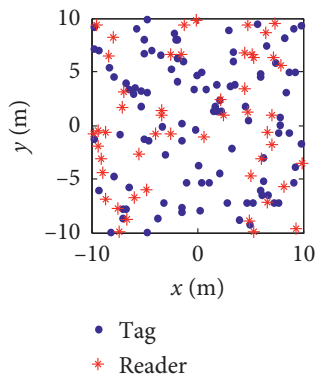

(g)

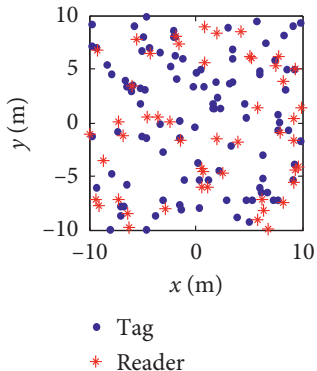

(c)

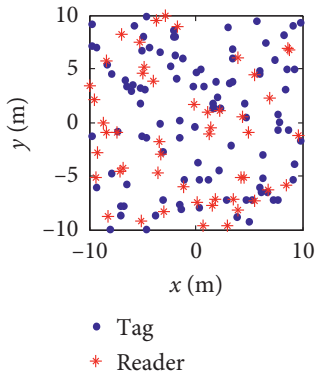

(h)

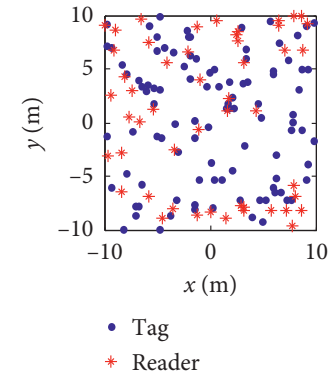

(d)

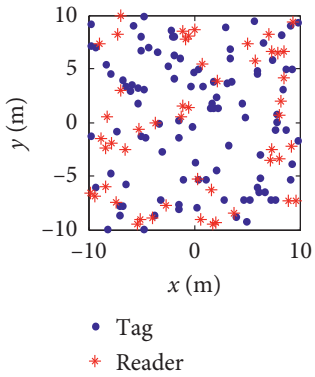

(i)

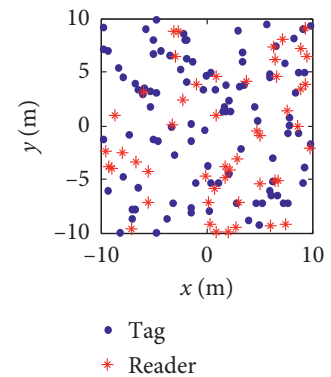

(e)

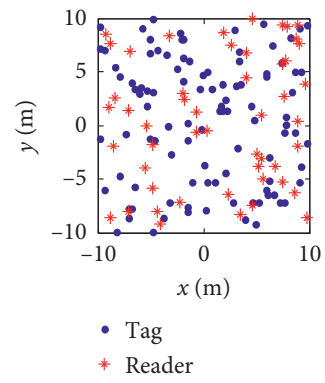

(j)

Figure 2: The initial position values of the 50 particles. (a) Reader 1. (b) Reader 2. (c) Reader 3. (d) Reader 4. (e) Reader 5. (f) Reader 6. (g) Reader 7. (h) Reader 8. (i) Reader 9. (j) Reader 10.

algorithm, which corresponds to 50 deployment scenarios. The initial position values of the 50 particles are shown in Figure 2.

In the 50 deployment scenarios, the best scenario is the $1^{\text {st }}$ through calculating the fitness value, which is shown in Figure 3 . The coordinates of the 10 readers are, respectively, $(-4.28,9.73),(-1.36,1.48),(0.54,-6.05),(-6.69,-0.02)$, $(9.80,-2.12),(4.28,-6.48),(-8.59,-6.85),(-6.77,-4.33)$, $(0.73,5.46)$, and $(7.61,2.75)$, and the function value is $51.6 \%$.

A random number between two $[-7,7]$ is randomly generated as the $X$-axis velocity and the $Y$-axis velocity of each deployment position, the initial velocity value of the particles in the particle swarm algorithm, and the initial velocity of the readers 1 to 6 is shown in Figure 4 .

4.2. Simulation Results and Analysis. The result of the RFID network planning simulation run once using standard PSO and DEEPSO is shown in Figures 5 and 6.

It can be seen from Figures 5 and 6 that the distribution of readers after optimization is more reasonable. The coordinates of the 10 readers by the standard PSO are, respectively, $(-4.96,9.52),(0.09,0.16),(-1.55,-5.49),(-5.21$, $-1.99),(9.93,-1.77),(5.02,-5.96),(-7.00,-9.30),(-5.13$, $-3.87),(-1.55,5.16)$, and $(8.27,5.71)$, the optimization function value is $79.2 \%$ when the generation value is 35 . The coordinates of the 10 readers by the DEEPSO are, respectively, (2.40, 4.45), (1.97, -1.2), (8.86, -3.15), (5.28, $-0.48),(0.32,8.20),(-0.59,-4.04),(-4.95,-7.87),(5.17$, $-7.47),(-5.26,5.24)$, and $(7.30,4.73)$, the optimization function value is $81.6 \%$ when the generation value is 119 . Compared with RFID networks planning using PSO, RFID

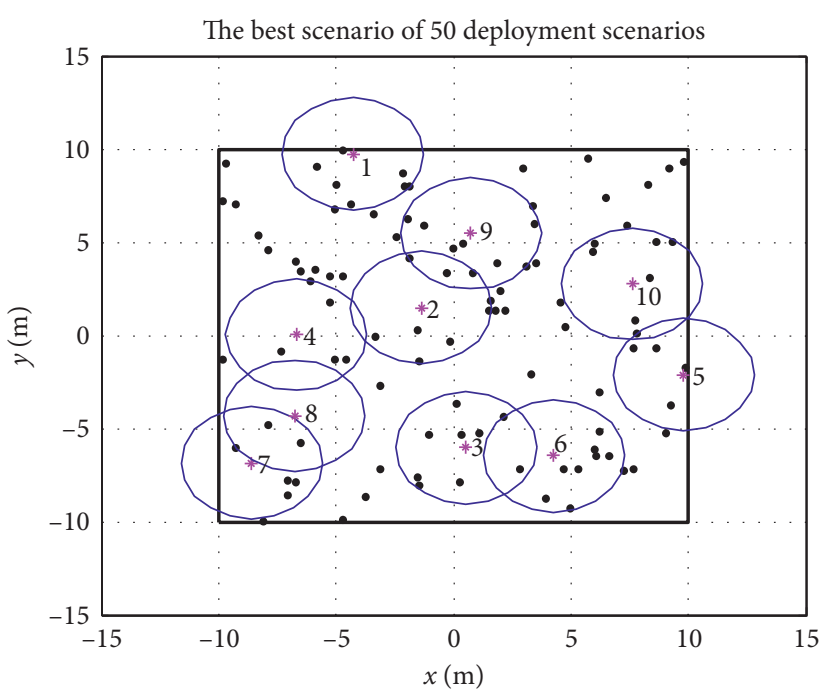

Figure 3: The best scenario of 50 deployment scenarios.

networks planning using DEEPSO shows the better optimization performance, which makes the function value increase by $2.4 \%$.

In order to further verify the optimization performance of the proposed method, the simulation experiment environment and parameters remain unchanged, 50 independent optimization experiments are performed respectively. The performance comparison of the two methods is shown in Figure 7.

It can be seen from Figure 7 that the min fitness value is $58.4 \%$, the max fitness value is $84 \%$, the mean fitness value is 


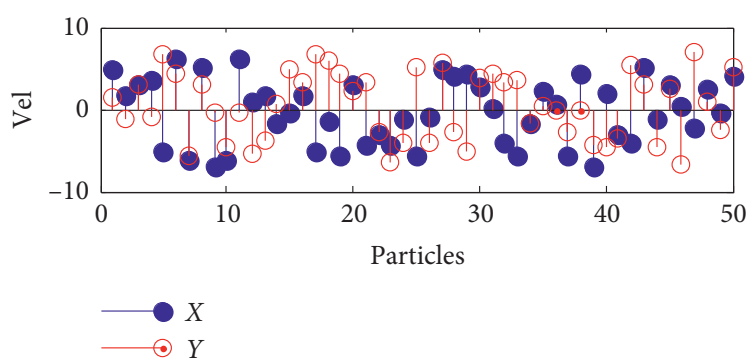

(a)
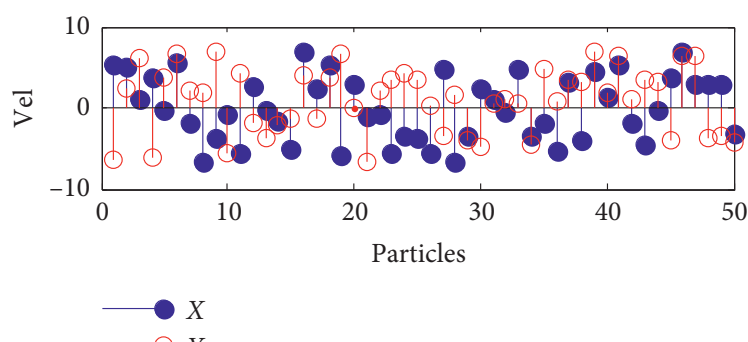

(c)

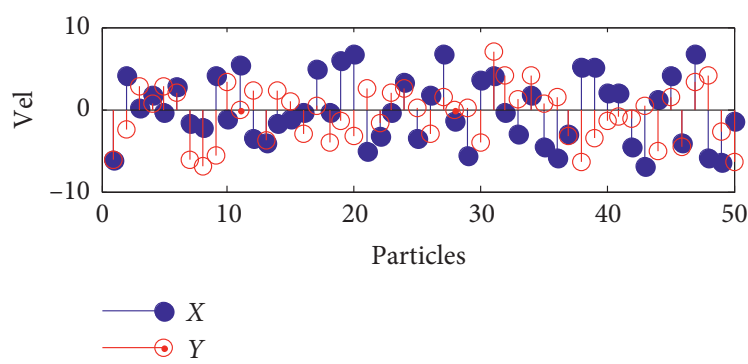

(e)

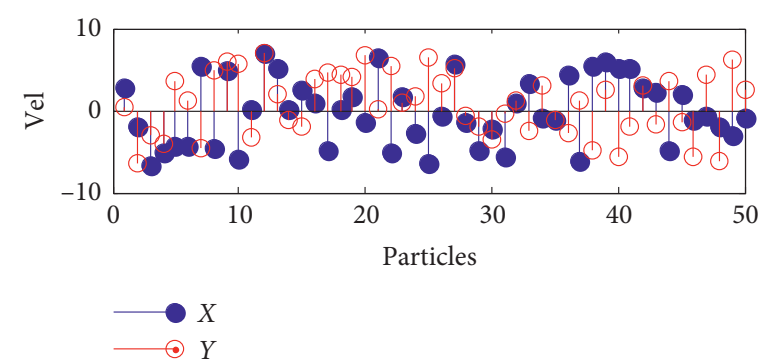

(b)

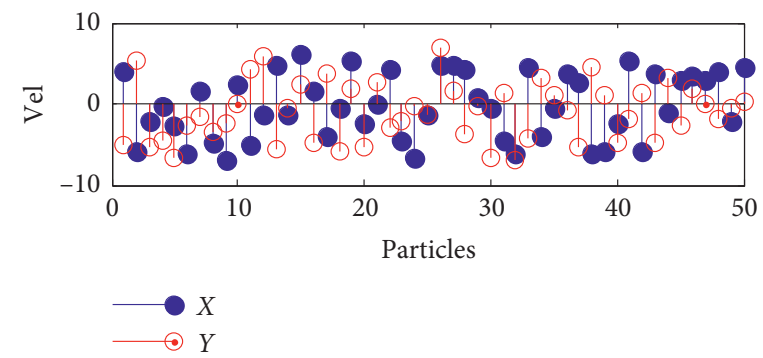

(d)

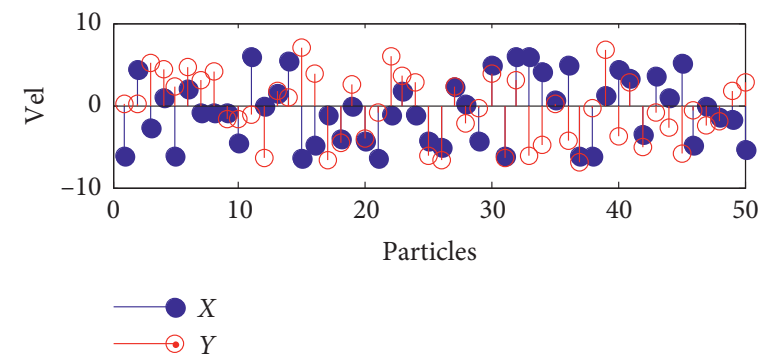

(f)

Figure 4: The initial velocity of the 50 particles in the readers 1 to 6. (a) Reader 1. (b) Reader 2. (c) Reader 3. (d) Reader 4. (e) Reader 5. (f) Reader 6.

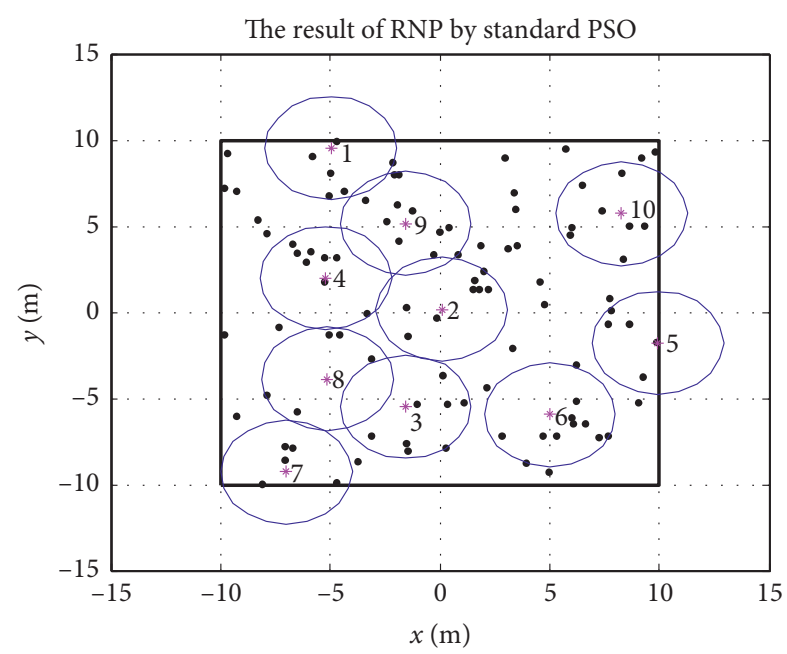

(a)

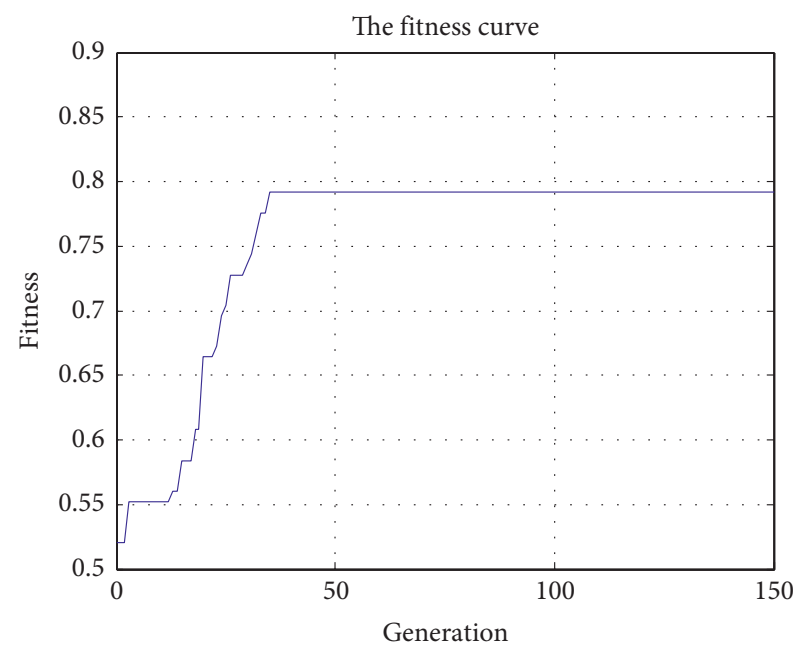

(b)

FIGURE 5: The simulation result of RFID network planning by the standard PSO.

$77.36 \%$ in RNP by PSO, and the min fitness value is $72 \%$, the max fitness value is $88 \%$, the mean fitness value is $80.75 \%$ in RNP by DEEPSO. Comparing with RNP by PSO, the mean fitness value of RNP by DEEPSO can be improved by $3.39 \%$. The simulation results show that the method of RNP by DEEPSO has the better optimization performance. This is 


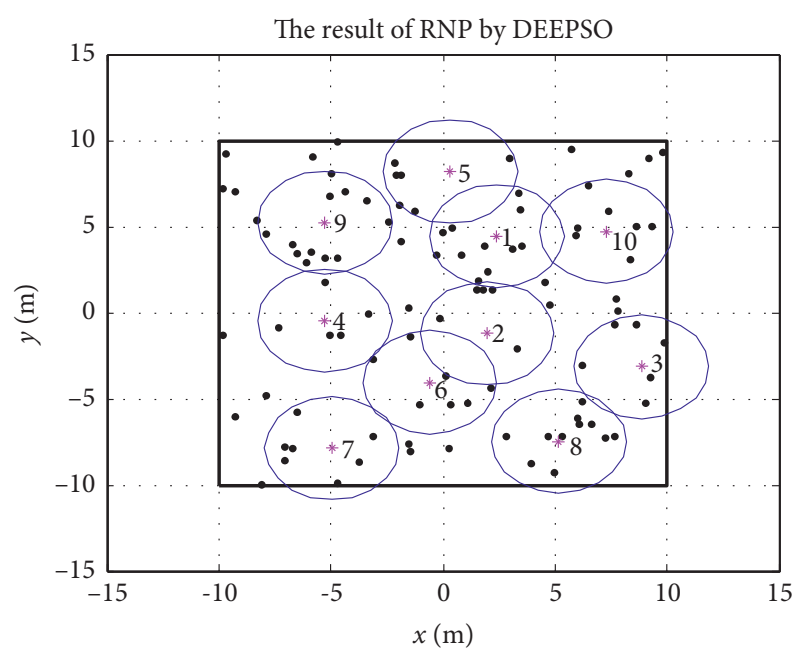

(a)

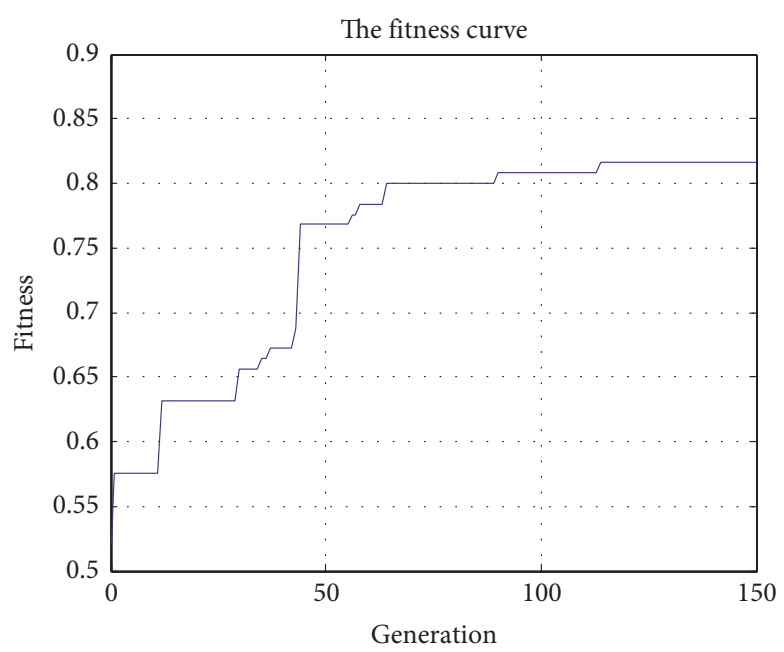

(b)

FIGURE 6: The simulation result of RFID network planning by DEEPSO.

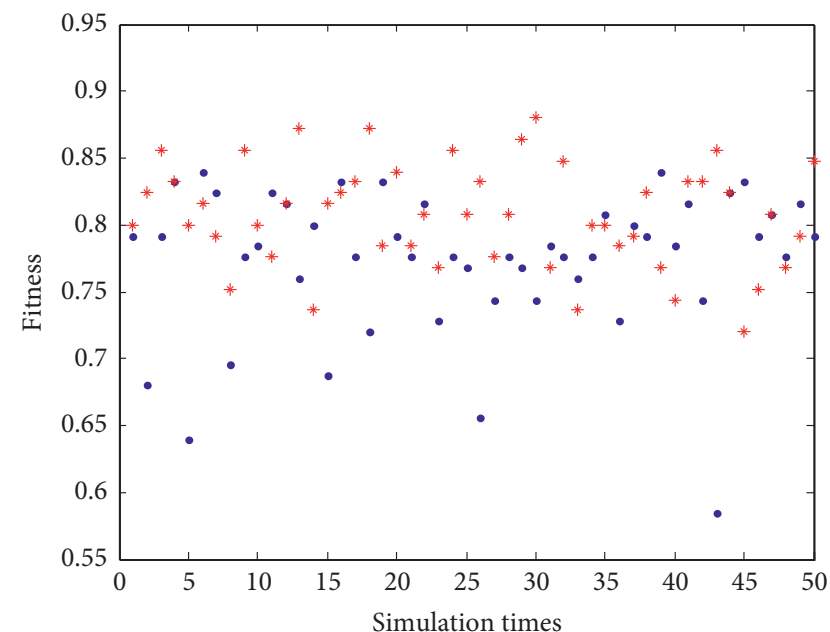

- RNP by Standard PSO

* RNP by DEEPSO

Figure 7: Comparison of two methods running 50 times.

because DEEPSO, which adds Differential Evolution (DE) and Evolutionary Strategies (ES) to the standard PSO, has continuous optimization ability, especially the later of the optimization process.

\section{Conclusions}

In this paper, aimed at solving the RFID networks planning problem, we present a mathematical model considering the tag coverage and the reader interference. In order to align with the same objective optimization direction, we present a transformed formula of the mathematical model as the fitness function. The DEEPSO algorithm that adds Differential Evolution (DE) and Evolutionary Strategies (ES) to the standard PSO is introduced to the optimization of RFID Networks Planning, which can improve the global convergence ability and particle diversity and can avoid falling into local convergence. According to the simulation results, compared with RFID networks planning by standard PSO, RFID networks planning by DEEPSO shows better optimization performance superior. Further research efforts should focus on the modeling and optimization of RFID network planning problem considering the adjustable radiated power of RFID reader.

\section{Data Availability}

The experiment data used to support the findings of this study were supplied by Hongshan Kong under license and so cannot be made freely available. Requests for access to these data should be made to m13643861930@163.com.

\section{Conflicts of Interest}

The authors declare that there are no conflicts of interest regarding the publication of this paper.

\section{Acknowledgments}

This research work was financially supported by the Fundamental Research Funds of Institute of Science and Technology (no. 2015603503).

\section{References}

[1] L. Ma, K. Hu, Y. Zhu, and H. Chen, "Cooperative artificial bee colony algorithm for multi-objective RFID network planning," Journal of Network and Computer Applications, vol. 42, pp. 143-162, 2014.

[2] L. Ma, H. Chen, K. Hu, and Y. Zhu, "Hierarchical artificial bee colony algorithm for RFID network planning optimization," The Scientific World Journal, vol. 2014, Article ID 941532, 21 pages, 2014.

[3] Y. Gao, X. Hu, H. L. Liu, and Y. Feng, "Multiobjective estimation of distribution algorithm combined with PSO for RFID network optimization," in Proceedings of International Conference on Measuring Technology and Mechatronics 
Automation (ICMTMA'10), vol. 2, pp. 736-739, Changsha, China, March 2010.

[4] S. Lu and S. Yu, "A fuzzy $k$-coverage approach for RFID network planning using plant growth simulation algorithm," Journal of Network and Computer Applications, vol. 39, pp. 280-291, 2014.

[5] Y. H. Yang, Y. J. Wu, M. Xia, and Z. J. Qin, "A RFID network planning method based on genetic algorithm," in Proceedings of the International Conference on Networks Security, Wireless Communications and Trusted Computing (NSWCTC'09), vol. 1, pp. 534-537, Wuhan, China, April 2009.

[6] H. N. Chen and Y. L. Zhu, "RFID networks planning using evolutionary algorithms and swarm intelligence," in Proceedings of the 4th International Conference on Wireless Communications, Networking and Mobile Computing (WiCOM'08), pp. 1-4, Dalian, China, October 2008.

[7] I. Bhattacharya and U. K. Roy, "Optimal placement of readers in an RFID network using particle swarm optimization," International Journal of Computer Networks \& Communications, vol. 2, no. 6, pp. 225-234, 2010.

[8] H. Kong and B. Yu, "An improved method of WSN coverage based on enhanced PSO algorithm," in Proceedings of IEEE Joint International Information Technology and Artificial Intelligence Conference (ITAIC2019), pp. 1294-1297, Chongqing, China, May 2019.

[9] N. Bacanin, M. Tuba, and R. Jovanovic, "Hierarchical multiobjective RFID network planning using firefly algorithm," in Proceedings of International Conference on Information and Communication Technology Research (ICTRC2015), pp. 282285, Abu Dhabi, UAE, May 2015.

[10] L. B. Ma, K. Y. Hu, Y. L. Zhu, and H. N. Chen, "Computational evolution of social behavior in bacterial colony optimization model," Journal of Pure \& Applied Microbiology, vol. 7, pp. 48-493, 2013.

[11] F. Han and J. Qi, "Optimal RFID networks planning using a hybrid evolutionary algorithm and swarm intelligence with multi-community population structure," in Proceedings of International Conference on Advanced Communication Technology (ICACT2012), pp. 1063-1068, Pyeong Chang, South Korea, February 2012.

[12] M. Tuba, N. Bacanin, and M. Beko, "Multiobjective RFID network planning by artificial bee colony algorithm with genetic operators," in Proceedings of 2015 International Conference in Swarm Intelligence (ICSI2015), pp. 247-254, Shanghai, China, June 2015.

[13] V. Miranda and R. Alves, "Differential evolutionary particle swarm optimization (DEEPSO): a successful hybrid," in Proceedings of the 2013 BRICS Congress on Computational Intelligence and 11th CBIC Brazilian Congress on Computational Intelligence, Ipojuca, Brazil, September 2013.

[14] L. M. Carvalho, F. Loureiro, J. Sumaili et al., "Statistical tuning of DEEPSO soft constraints in the security constrained optimal power flow problem," in Proceedings of International Conference on Intelligent System Application to Power Systems (ISAP2015), Porto, Portugal, September 2015. 


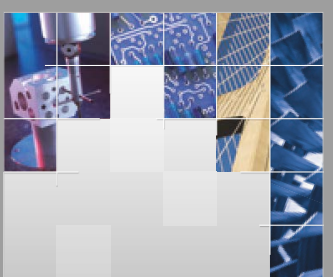

\section{Enfincering}
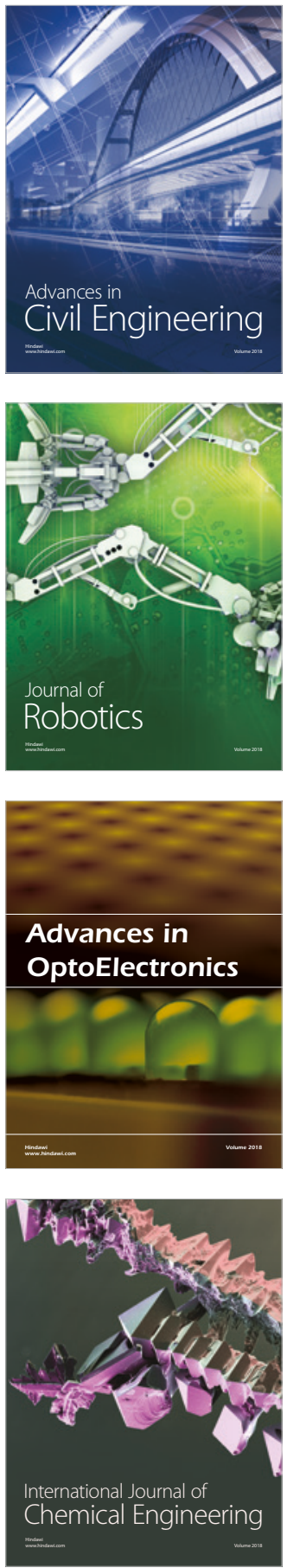

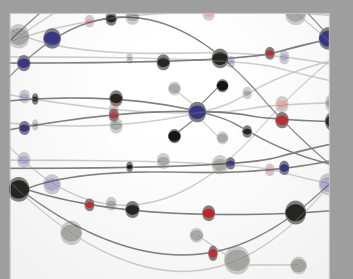

\section{Rotating \\ Machinery}

The Scientific World Journal

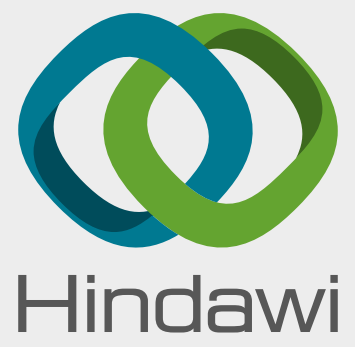

Submit your manuscripts at

www.hindawi.com
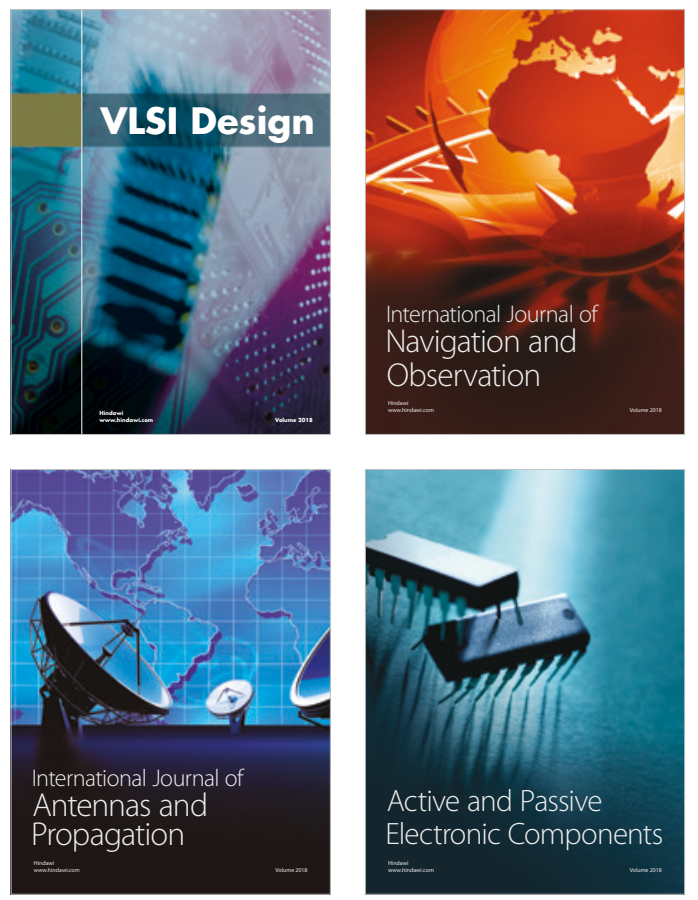
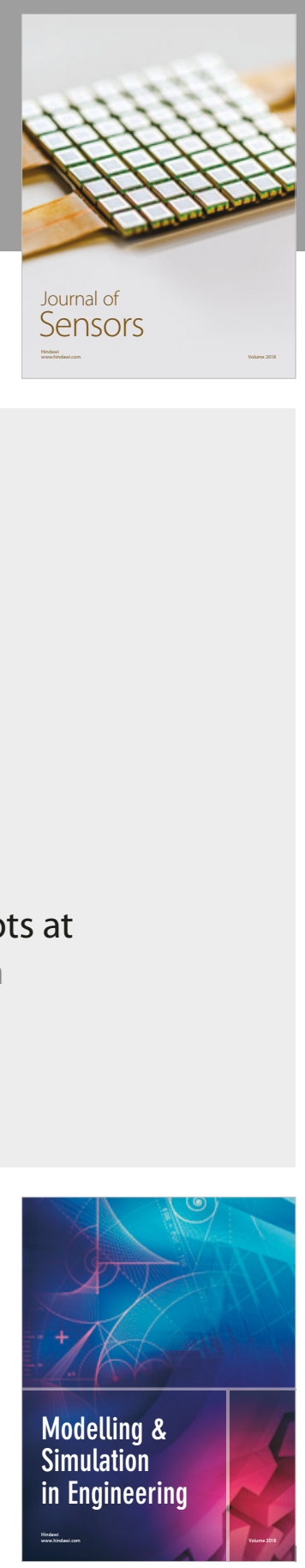

\section{Advances \\ Multimedia}
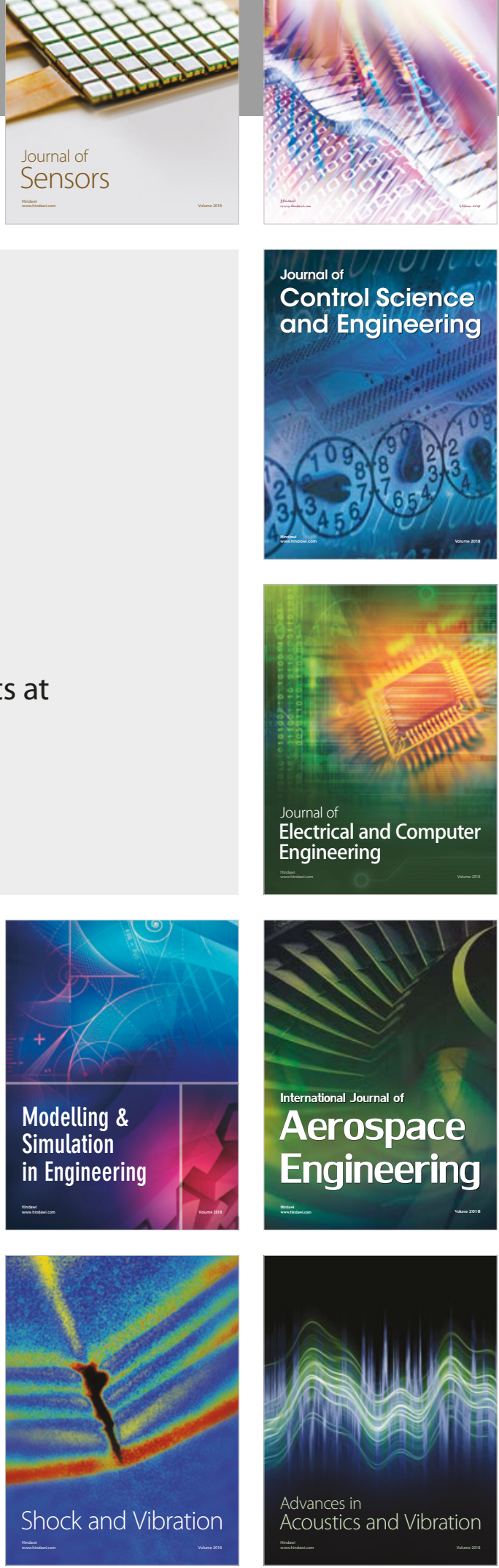